\title{
HISTORIA DE LAS EMOCIONES: UNA CORRIENTE HISTORIOGRÁFICA EN EXPANSIÓN
}

\author{
Juan Manuel Zaragoza Bernal \\ Centro de Ciencias Humanas y Sociales - CSIC \\ jm.zaragozabernal@gmail.com
}

Recibido: 2 febrero 2012; Aceptado: 7 octubre 2012.

Cómo citar este artículo/ Citation: Zaragoza, Juan Manuel (2013), "Historia de las emociones: una corriente historiográfica en expansión", Asclepio 65 (1): e012. doi: http://dx.doi.org/10.3989/asclepio.2013.12

Copyright: (c) 2013 CSIC. Este es un artículo de acceso abierto distribuido bajo los términos de la licencia Creative Commons Attribution-Non Commercial (by-nc) Spain 3.0.

\section{INTRODUCCIÓN}

Peter Burke, en un texto publicado en 2005, se preguntaba si existía una historia cultural de las emociones (Burke, 2005, p. 35-48). La pregunta resulta cuanto menos extraña teniendo en cuenta que dicho texto se encuentra en un libro que, explícitamente, se catalogaba como "historia de las emociones" (Gouk y Hills, 2005b). Las editoras del libro - una, historiadora de la medicina, la otra, del arte-, tomaban como punto de partida del mismo, precisamente, la historicidad de las emociones, no sólo de sus concepciones y representaciones, sino de las emociones mismas ${ }^{1}$. Tras hacer un breve análisis de la producción historiográfica que, de una forma u otra, ha tratado el tema de las emociones, Burke concluye que todas ellas acarrean el mismo pecado: la falta de un marco analítico riguroso. Es decir, una falta de acuerdo en cómo entendemos nuestro objeto de estudio (¿Hablamos de emociones o de afectos? ¿Qué es una emoción? ¿Lo es la ira? ¿Lo es la fraternidad? ¿Estudiamos emociones o representaciones de emociones?); de quién son las emociones que estudiamos o debemos estudiar (¿hombres, mujeres? ¿Jóvenes, viejos?); con qué métodos, conceptos y teorías debemos aproximarnos a esta nueva historia; y, por último, qué fuentes deben o pueden utilizarse para este estudio (Burke, 2005, p.
38-39). Lo que Burke viene a decir, a fin de cuentas, es que aunque muchos autores hayan historiado las emociones, todavía no se ha hecho una historia de las emociones, puesto que más allá de unos compromisos mínimos (como no problematizar la definición de emoción), no se ha desarrollado una disciplina (o subdisciplina) con todo lo que ello conlleva: unos métodos propios, unas fuentes específicas, un objeto de estudio bien definido, etc.

Desde finales de los años 90 del siglo XX, pero sobre todo en la primera década del siglo XXI, los textos dedicados al estudio de la historia de las emociones se han multiplicado. Muchos de ellos han dedicado gran parte de sus esfuerzos a encontrar precedentes, a realizar una genealogía del estudio histórico de las emociones que sirva de punto de partida a sus propios estudios. Estas genealogías coinciden en señalar una serie de nombres que, de una forma u otra, pueden considerarse precursores de este nuevo interés en las emociones como objeto histórico. Están, obviamente, los nombres clásicos señalados por Burke (Nietzsche, Huizinga, Febvre, Elias), junto a otros muchos procedentes de la escuela de Annales (Braudel, Ariès, Chartier), e historiadores americanos de la corriente llamada emocionología, principalmente Peter N. Stearns y Carol Z. Stearns ${ }^{2}$. Y pese a todo, pese a esta cadena de 
antecesores ilustres, todos los autores de estos textos comparten el análisis final de Burke: nunca, hasta este momento, se ha hecho historia de las emociones. Las razones que se aducen son variadas: la misma naturaleza de la disciplina, prisionera de una suerte de pecado original que, a través de la servidumbre política que encontramos en su origen, la conduce hacia intereses "racionales" (Rosenwein, 2002, p. 821); una falta de "foco", debido, principalmente, a la "invisibilidad" de los sentimientos subjetivos, pero también a la propia indefinición de qué es una emoción (Bourke, 2003, p. 114); la amplia comprensión de "las emociones" como parte de la naturaleza humana y que, por tanto, no tendrían historia (Gouk y Hills, 2005, p. 16); o el interés, tal vez exagerado, en los procesos de cuantificación de emociones (Alberti, 2006, p. xv). Sea cual sea la razón esgrimida, el resultado es siempre el mismo: no hay, hasta el momento, ninguna historia de las emociones. $\mathrm{O}$ al menos, una historia de las emociones "correcta". Esta postura más matizada es la que sostiene Rosenwein, que inicia su libro sobre comunidades emocionales declarando, por un lado, la antigüedad del tema -"historians has always taIked about emotions" (Rosenwein, 2006, p.1)-, para, en la frase posterior, denunciar la incorrección de sus planteamientos, incorrección que es una de las causas que empujan a la autora a escribir su libro: "The fact that there is a history of emotions but that it has been studied (for the most part) wrongly or badly is one reason that I have written this book." (Rosenwein, 2006, p. 1).

Es esta situación de "novedad absoluta" la que convierte cada libro, cada artículo publicado, en un intento de sentar las bases necesarias para que esa posible historia de las emociones se escriba "correctamente". La comprensión de su originalidad, sin embargo, no ha ayudado a que la historia de las emociones supere la situación en que Burke la encontró en 2005: falta de un marco analítico riguroso. En el siguiente apartado intentaremos, a partir del esquema aportado por Burke, analizar algunas de dichas propuestas. Para que dicho análisis sea manejable, sin embargo, quedará restringido a las obras publicadas a partir del año 2000, momento en que hemos fechado este "resurgir" de la historia de las emociones.

\section{ACUERDOS Y DESACUERDOS EN LA HISTORIA DE LAS EMOCIONES}

\subsection{Afectos, emociones, representaciones}

Si tuviéramos que señalar una debilidad de la historia de las emociones, y sólo una, esa sería, sin dudarlo un segundo, la ausencia de una definición adecuada de su objeto de estudio, empezando por su nombre: ¿emociones o afectos? (Labanyi, 2010) ¿O tal vez sensibilidades? (Wickberg, 2007) ¿Debemos prestar atención a lo que la psicología nos dice acerca de las emociones 0 , por el contrario, debemos dejarlo de lado? (Richards, 2005) ¿Debemos centrarnos en las representaciones de las emociones (Gouk y Hills, 2005a, p. 26), en los cambios en el lenguaje (Dixon, 2003), en narraciones explícitamente sobre emociones (Rosenwein, 2010, p. 12-14)? ¿O, por el contrario, deberemos ir más allá de los textos directamente relacionados con las emociones y buscar una suerte de "sensibilidad" más amplia (Wickberg, 2007, p. 662)? Como bien decía Burke, en su ya citado capítulo, el único consenso es no problematizar en exceso la definición del objeto de estudio.

Y sin embargo, muchas de las preguntas que hemos formulado en el párrafo anterior son la consecuencia directa de esa falta de problematización, más concretamente de la negativa a decidirse entre dos posibles definiciones de emociones: una que afirma que son hechos naturales y, por lo tanto, carentes de historia y otra que afirma todo lo contrario, que son constructos sociales sujetos a variaciones históricas. En definitiva, la vieja disputa entre cultura y naturaleza (Alberti, 2006, p. xvii), un debate tal vez heredado de las principales disciplinas que han dado forma a este nuevo interés en las emociones: la antropología, por un lado, y la psicología por el otro (Reddy, 2001). Las respuestas varían de un autor a otro, pero en pocos casos se trata de respuestas amplias, que vayan más allá de declaraciones programáticas en una u otra dirección:

There is, therefore, no overarching definition of 'emotions' that applies to all periods and all places; nor would a list enumerating specific emotions (fear, anger, and so on) serve to explain how such terms were conceived, and how these constructs were employed. Nevertheless, precisely because emotions are culturally mediated and, therefore, culturally variable, an analysis of the ways in which they were defined, by whom, and for what purposes is an important undertaking. (Gouk y Hills, 2005a, p. 16)

Esta afiliación al "construccionismo" es mayoritaria. No podría ser de otra forma, dado el peso que el postmodernismo tiene en la historia cultural contemporánea. Pero es que, como Jordanova declara de forma contundente, esta aproximación es la única posible si queremos tener una visión amplia de los procesos históricos que incluya ideologías, ideas, imágenes y cultura material (Jordanova, 2004, p. 356), es decir, si realmente queremos hacer historia cultural. Obviamente, esta opción entraña sus riesgos y si bien, como señala Burke, los estudios realizados desde esta perspectiva son "más innovadores" el precio a pagar es que sus conclusiones son "más difíciles de sostener" (Burke, 2006, p. 136). Por el contrario, las que se decantan por la otra opción son, en su opinión, no sólo menos innovadoras, sino que al constreñir sus estu- 
dios a las actitudes conscientes ante las emociones lo que obtienen no es una historia de "las propias emociones", sino una "historia intelectual" (Burke, 2006, p. 136; Burke, 2005, p. 40), por muy sólida que pueda llegar a ser.

Ahora bien, ¿es posible escapar de esta dicotomía? ¿Debemos, incluso si partimos de una posición construccionista, tener en cuenta lo que la psicología nos dice sobre las emociones? Una crítica que se desprendía del ya citado texto de Wickberg sobre la historia de las sensibilidades era que la historia de las emociones no tenía en cuenta el papel cognitivo de las emociones, señalado por ciertas corrientes de la psicología, y que por tanto trataba las emociones como "a discrete realm rather than seeing them as linked to larger characteriological patterns involving modes of perception and thinking as well as feeling" (Wickberg, 2007, p. 682). En una carta al editor de The American Historical Review, Rosenwein polemizaba con Wickberg precisamente sobre este punto, ya que, según ella, historiadores como Peter N. Stearns, William M. Reddy o ella misma defienden la intrínseca relación entre emoción y cognición, siendo la primera una modalidad de la segunda (Rosenwein, 2007, p. 1313). En opinión de estos autores, incluso si tomamos una posición constructivista, las corrientes en psicología que relacionan emoción y cognición son relevantes para su labor histórica ${ }^{3}$. Sin duda el más ambicioso de los defensores de esta posición es William M. Reddy.

Reddy parte de una crítica que podemos calificar de política, incluso ética, hacia el constructivismo. Sin negar lo positivo de esta corriente, Reddy (2001, p. 54) denuncia la incapacidad de criticar, desde sus postulados, las prácticas locales que estudia. Una vez que hemos identificado un determinado conjunto de prácticas culturales como opresivas, imperialistas, machistas o racistas, no tenemos, sin embargo, ningún criterio para criticarlas ya que, localmente, y en tanto que toda opción es una "construcción", ¿cómo podemos preferir una sobre otra (Reddy, 2001, p. 74)? Reddy no es el único que señala esta incapacidad política del construccionismo (Fissell, 2004, p. 384-285), si bien en su caso la necesidad de superar esta limitación es más acuciante, puesto que pretende sentar las bases para poder realizar una crítica de los diversos regímenes emocionales teniendo en cuenta el mayor o menor grado de "libertad emocional" que permiten:

We need a conceptual frame that acknowledges the importance of management (as opposed to construction) of emotion, that allows political distinctions among different management styles on the basis of a concept of emotional liberty, and that permits the narration of significant historical shifts in such management styles (Reddy, 2001, p. 118).

El marco que propone Reddy se basa, por un lado, en una teoría de la "traducción" que se presenta como una respuesta al postulado derridiano del "no hay fuera de texto" (Derrida, 1976, p. 158), esto es, la imposibilidad de alcanzar el "significado desnudo", de escapar de la tiranía de los significantes (Reddy, 2001, p. 76). La teoría de la traducción de Reddy pretende minimizar, cuando no eliminar, la distancia entre langue y parole, al entender al individuo como receptor de múltiples mensajes cifrados en diversos códigos que son traducidos, a veces con éxito, otras veces sin él, a códigos más "manejables" (Reddy, 2001, p. $80)$. Este proceso de traducción se relaciona estrechamente con dos elementos derivados de la psicología cognitiva, el de "activación" y el de "atención". Con el primero, Reddy señala aquellos elementos que ponen en marcha nuestra maquinaria cognitiva (se identifiquen como "inputs", "pensamientos", "memorias", etc., que Reddy resume con la expresión "thought material"). Sin embargo, no todas las activaciones despiertan nuestra atención -el "lugar" en que el proceso de traducción entre códigos se produce, dando lugar a frases declarativas o actos intencionales-, sino que quedan a un nivel no consciente (Reddy, 2001, p. 88-89). Podemos entender el interés que este tipo de estados tiene para una teoría de las emociones, que serían entendidas como "activaciones" que no llegan a llamar nuestra "atención", debido a que están relacionadas con redes de objetivos complejos, pertenecientes a diversos códigos y agrupados en esquemas, que requieren de traducción, y que exceden nuestra capacidad de atención en el corto plazo, debido, precisamente, a la dificultad de dicho proceso (Reddy, 2001, p. 94). Esta conceptualización de las emociones daría lugar a una nueva concepción del yo, que escaparía de dualismos cartesianos pero también de los sujetos imaginarios del post-estructuralismo. Un yo "desagregado", sin una unidad inherente, derivado de su constante interacción con múltiples flujos de significantes, procedentes de diversos códigos, que deben ser traducidos. Un yo que es social, en tanto que los procesos de integración del yo (la traducción de los "significantes" en actos intencionales) se construye sobre la interacción social, pero no construcciones colectivas (Reddy, 2001, p. 95).

El siguiente eslabón en el marco que nos propone Reddy, cuyo objetivo, recordamos, era encontrar una forma de "evaluar" regímenes emocionales, consiste en desarrollar una teoría de las expresiones emocionales como actos de habla, actos que denomina emotives, y que vendrían a ser el proceso de traducción más o menos provisional de todas esas activaciones. Basándose en la definición de Austin de "enunciado performativo", Reddy sostiene que los enunciados sobre emociones (del tipo "estoy enfadado") tienen una función descriptiva (de cómo me siento), relacional (al presentar una condición a la interacción) y autoexploratoria (porque tal vez no estoy enfadado, sino 
triste), así como un efecto modificador (al decantarme por una opción, estoy reafirmando una traducción entre las posibles, modificando mi cadena de objetivos y mis relaciones con ella) (Reddy, 2001, p. 100-105).

Un régimen emocional estaría formado por las normas que permiten manejar estos emotives, es decir, qué emotives son lícitos y cuáles no en una situación determinada, ante una persona concreta, etc. Estos regímenes tienen consecuencias para la construcción del individuo, pues, debido a su poder exploratorio, la posibilidad de expresar o no ciertos emotives se convierte en la posibilidad de explorar cadenas de objetivos hasta ese momento más allá de nuestra atención, lo que Reddy llama navegación emocional: la posibilidad de explorar nuevas posibilidades y cambiar nuestros objetivos, lo que constituiría, en última instancia, una definición de libertad emocional:

The idea of navigation as a universal, central characteristic of emotional life makes possible a preliminary definition of 'emotional liberty' as the freedom to change goals in response to bewildering, ambivalent thought activations that exceed the capacity of attention and challenge the reign of high-level goals currently guiding emotional management. This is freedom, not to make rational choices, but to undergo conversion experiences and life-course changes involving numerous contrasting, often incommensurable factors (Reddy, 2001, p. 122-123).

Un régimen emocional será estricto o tolerante dependiendo del grado de libertad que permita, de los emotives que puedan ser utilizados, de las rutas de navegación que estén abiertas. El primero, al restringir las condiciones de autoconocimiento y las elecciones vitales, provoca un sufrimiento emocional -un conflicto de objetivos (Reddy, 2001, p. 123)- en el individuo, permitiéndonos, de esta forma, distinguir entre regímenes justos e injustos (Reddy, 2001, p. 128-130). La pregunta a la que deberemos enfrentarnos, según Reddy, es ¿quién sufre?

This array of concepts frees one of the necessity of theorizing culture, power, or identity characteristics, such as race, class, gender, or ethnicity, which have preoccupied scholars so much of late. The only questions that need to be asked are, Who suffers? Is the suffering an unavoidable consequence of emotional navigation or does this suffering a tragedy or an injustice? (Reddy, 2001, p. 130)

Como hemos indicado, el texto de Reddy es el más ambicioso de entre todos los escritos hasta el momento en la historia de las emociones. Su intención es ofrecernos un marco de análisis que, partiendo de los hallazgos más interesantes en los campos de la psicología y la antropología, permita superar las limitaciones que el construccionismo impone al historiador. Se trata de una apuesta valiente, y como tal suge- rente. Algunos de los conceptos acuñados por Reddy (sufrimiento emocional, refugio emocional, etc.) son herramientas útiles para la tarea de estudiar las emociones del pasado, y su enfoque, optimista con las oportunidades que se presentan para el ejercicio de la agencia, incluso en las situaciones más restrictivas, y políticamente comprometido, no deja de ser inspirador. Sin embargo, como toda propuesta que pretende ser innovadora no escapa a las críticas, algunas de calado. La principal tiene que ver con el concepto de libertad empleado, que debe demasiado a la tradición liberal de occidente y su sujeto auto-contenido, así como a sus prácticas constitutivas, sobre todo a una introspección propia de las clases medias y altas de occidente a partir del siglo XIX (Nye, 2003, p. 923). Por otra parte, el énfasis en la expresión de las emociones a través de los emotives nos hace preguntarnos cómo podemos investigar las emociones de aquellos cuyos emotives no han sido registrados, de aquellos que nunca nos dejaron un enunciado emocional. Una historia de este tipo debería limitarse, por tanto, a las élites europeas ilustradas. Por otra parte, al final, y pese a todo, el libro de Reddy nos dice que la historia de las emociones es la historia de las normas que las gestionan, tal y como, según Burke, debemos esperar de aquellos libros que no apuestan por la historicidad de las emociones, sino tan sólo por sus "modulaciones".

En resumen, vemos que no se ha hecho un esfuerzo por definir de forma más o menos precisa qué son las emociones y, cuando así se ha intentado, los resultados no han sido plenamente satisfactorios, al anclar dicha definición en unas nociones de sujeto y de libertad occidentales. Lo que encontramos en la mayoría de los textos, por tanto, es una comprensión de las emociones más o menos "popular" (como la define el diccionario de la RAE: alteración del ánimo intensa y pasajera, agradable o penosa, acompañada de cierta conmoción somática), señalando, en algunos casos, su relación con los procesos de cognición sin que ello tenga ningún tipo de incidencia en la historia que se narra ${ }^{4}$. El ejemplo de Reddy nos lleva a pensar, sin embargo, que una mejor definición de qué sea una emoción y cuáles sus funciones puede resultar productiva a la hora de plantear nuevas alternativas de estudio en la historia de las emociones.

\section{2. ¿Quién se emociona?}

Burke señalaba como segundo elemento a mejorar lo que él llama una "sociología" de las emociones, esto es, ¿quién se emociona? No se trata, en absoluto, de una cuestión baladí, pues, como hemos visto en el caso de Reddy, el no hacernos esta pregunta puede conducirnos a que la historia narrada sea la historia de una minoría. El concepto de "comunidad emocional", desarrollado por Barbara Rosenwein, pretende ser una respuesta a esta pregunta, por mucho que ella 
no lo plantee en estos términos. Rosenwein define el concepto de "comunidad emocional" como:

[...] groups in which people adhere to the same norms of emotional expression and value - or devalue - the same or related emotions. More than one emotional community may exist -indeed normally does existcontemporaneously, and these communities may change over time (Rosenwein, 2006, p. 2). ${ }^{5}$

Al centrar nuestra atención en las comunidades, Rosenwein nos pide, en primer lugar, que identifiquemos quiénes son los miembros de la misma, esto es, quiénes comparten estas normas y valoran emociones similares. Solventamos de esta forma la cuestión sobre el quién, pero surgen nuevos problemas. El primero, y tal vez más evidente, es la posibilidad de que una persona pertenezca a más de una comunidad, incluso al mismo tiempo. Esta posibilidad, ya contemplada por Fleck al hablar de los colectivos de pensamiento (Denkkollektiv) (Fleck, 1986, p. 157), introduce un dinamismo que explica el cambio de los colectivos de pensamiento y que creemos puede ayudar a explicar el cambio en las comunidades emocionales de Rosenwein. Tal vez a consecuencia de esta ausencia de dinamismo interno, el cambio en los casos estudiados por Rosenwein es siempre de una comunidad por otra. Nunca se explica el cambio dentro de una misma comunidad y, cuando se hace, se opta por una explicación claramente insatisfactoria, como la empleada para dar cuenta de las diferencias existentes entre las inscripciones de dos cementerios situados en la ciudad francesa de Trier entre los años 480 y 750 :

Were there two emotional communities at Trier, one using the northern cemetery, the other the southern? It seems unlikely. As we shall see, in both places the epitaphs emphasized family relationships, whether or not the explicitly expressed feelings. The differences between the 'emotional styles' of the cemeteries seem best explained by changes over time [...] I suggest that the Trier epitaphs from both northern and southern cemeteries were the product of one community that underwent gradual transformation over time in tandem with changes in cultic practices (Rosenwein, 2006, p. 65-66).

Resulta cuanto menos curioso explicar el cambio histórico apelando al paso del tiempo, pero esto no hace más que señalar el problema de fondo de una definición de comunidad emocional demasiado estática, en la que Rosenwein sugiere tránsitos de individuos de una comunidad a otra (Rosenwein, 2006, p. $25)^{6}$, sin que ello tenga mayor impacto en el interior de la comunidad. Problema derivado de una identificación excesiva entre "comunidades emocionales" y "comunidades sociales" (familias, monasterios, cortes principescas) (Rosenwein, 2010, p. 11). Las comunidades que nos presenta Rosenwein son, por tanto, pequeñas, cerradas y homogéneas en su com- posición. Tampoco ofrece ninguna explicación sobre su aparición, es decir, sobre cómo y por qué se crean comunidades emocionales. Al identificarlas con "comunidades sociales" pareciera darse por sentado que toda comunidad social es una comunidad emocional, explicándose la aparición de esta última por la de la primera (la creación de una nueva corte explicaría la aparición de una nueva comunidad emocional). Hasta qué punto estos problemas son el resultado de las fuentes disponibles para el periodo que estudia es algo que debatiremos en un apartado posterior.

Pese a estas debilidades, el gran mérito de la aproximación de Rosenwein consiste, precisamente, en señalar el papel social de las emociones como creadoras de comunidades. No sólo responde a la pregunta “¿quién se emociona?", sino que coloca esta identificación en primer plano. A partir de este estudio deberemos tener en cuenta que hablar de emociones es hablar siempre de individuos, aunque no exclusivamente de ellos.

\subsection{Conceptos, métodos y teorías}

El siguiente punto sobre el que Burke llama nuestra atención es el de los conceptos, métodos y teorías. Como hemos visto en los dos apartados anteriores, gran parte del trabajo realizado por los historiadores de las emociones ha consistido en desarrollar nuevos conceptos que les permitan trabajar con su novedoso objeto de estudio. Ya sean comunidades emocionales, estilos (Stearns, 1994), regímenes, o refugios, la tarea de acuñar términos que nos permitan hablar de las emociones en la historia es una de las tareas más fructíferas e interesantes, por lo que conlleva de experimentación y creatividad. No ha sido así, sin embargo, en el apartado metodológico. Esto es especialmente evidente en un reciente texto, ya citado, de Barbara Rosenwein titulado "Problems and Methods in the History of the Emotions". El mayor problema de Rosenwein, en este y otros textos, es la deuda que ha contraído con uno de sus principales referentes, que no es otro que Brian Stock y su análisis de las "comunidades textuales" (Rosenwein, 2010, p. 11)7. La propuesta de Rosenwein podría resumirse en el emplazamiento de estas "comunidades textuales", mediante la localización de textos relacionados con emociones (y sólo emociones) (Rosenwein, 2007, p. 1313), a través de la presencia, o no, de términos que las designen. Estos términos deberán ser problematizados (una palabra que nosotros relacionamos con una emoción puede estar relacionada con otra en el pasado) a través de la lectura de teóricos contemporáneos a la comunidad objeto de estudio y evaluando su importancia o "peso" en los textos (entendido, en primer lugar, como la frecuencia de aparición del término en el total del texto) (Rosenwein, 2010, p. 12-17). A estas recomendaciones une la de prestar atención a las pa- 
labras no dichas, las metáforas y las ironías. El método de Rosenwein, por muy novedoso que sea su objeto, no deja de ser el tradicional de la historia: localiza los textos pertinentes, analízalos y obtén conclusiones. Que la historia nace con la escritura es parte de la definición más tradicional de la disciplina. Que podemos ir más allá de ella es algo que hemos aprendido hace relativamente poco.

El libro editado por Gouk y Hills es, en este aspecto, bastante más interesante. Las aportaciones de los distintos autores van desde la historia de las ideas (Hills, 2005) hasta los estudios centrados en las prácticas científicas del siglo XX (Dror, 2005), sin olvidar el que tal vez sea el más interesante de todos los textos reunidos en este libro conjunto: el de Michael Schwartz y su estudio sobre Giotto y Piero de la Francesca, que intenta cambiar nuestra concepción sobre los affetti en la Baja Edad Media e inicios del Renacimiento a través del estudio de la cultura visual de estos autores (Schwartz, 2005). Lo mismo podemos decir del libro colectivo editado por Alberti y dedicado a la historia de la medicina (Alberti, 2006b), en el que el estudio de los cambios producidos en la terminología científica sobre emociones (Dixon, 2006) convive con la atención prestada a prácticas culturales más amplias, como las del mundo del espectáculo (Hayward, 2006). En este sentido, sin embargo, el ejemplo más interesante es, sin ninguna duda, el libro que Joanna Bourke (2005) dedica al miedo. Las prácticas y enfoques analizados por la autora son tan numerosos (desde el psicoanálisis al control de masas, del cuidado de los niños a las tecnologías de la información) que es fácil clasificar este libro, centrado en la cultura anglosajona desde mediados del siglo XIX hasta finales del $\mathrm{XX}$, como el mejor ejemplo posible de lo que Clifford Geertz llamó descripción densa. A la densidad descriptiva une Bourke su inteligente análisis crítico de otras aproximaciones posibles, como la emocionología y la psicohistoria, así como una reflexión sobre los límites de sus propias elecciones metodológicas ${ }^{8}$. Su propuesta para analizar la historia de las emociones, que pretende evitar algunas de las limitaciones ya expresadas en anteriores apartados de este ensayo, apuesta por centrar la atención en lo que la emoción (el miedo en su caso) hace (Bourke, 2005, p. 353), lo que nos reconduce, nuevamente, a la dimensión política de las emociones, su papel en las relaciones interpersonales y su nexo con el poder, una propuesta que ella llama Aestesiología (Bourke, 2005, p. 353-356; Bourke, 2003, p. 123-126).

Al hablar de teoría, por tanto, podemos detectar nuevamente el peso del construccionismo y la crítica post-moderna a la historia más tradicional en la atención prestada a prácticas heterogéneas, a las relaciones de poder, etc., sin dejar, por eso, de denunciar los límites de la post-modernidad. También hemos señalado el intenso trabajo de acuñación de conceptos, muchos de ellos compartidos por diversos autores. Sin embargo, al centrarnos en la metodología el resultado es bastante más decepcionante: el análisis de textos es el recurso más utilizado por los autores estudiados, excepto contados casos.

\subsection{Fuentes}

A la hora de tratar un nuevo tema de investigación histórica es fundamental realizar un profundo trabajo de revisión sobre las fuentes. Sin embargo, cuando no sólo el tema sino el enfoque del mismo comparten esa novedad, el trabajo sobre las fuentes se convierte en doblemente importante, y por tanto doblemente problemático (Burke, 2003). Ante tal situación el trabajo con las fuentes es doble: por un lado, la lectura de fuentes conocidas desde el nuevo punto de vista; por otro, la aportación de nuevas fuentes. La historia de las emociones ha sido especialmente fructífera en la primera de estas tareas. Un ejemplo evidente es el de William Reddy, que centra la segunda parte de su libro en una relectura de las fuentes conocidas sobre la revolución francesa (Reddy, 2001, p. 141-210), así como en el análisis de una serie de registros judiciales posteriores (Reddy, 2001, p. 211-314), todo ello desde el punto de vista de su teoría de las emociones. En la misma dirección se mueve Bourke, si bien en su caso, como ya hemos indicado, las fuentes utilizadas son tan numerosas como heterogéneas. Son especialmente inspiradores los capítulos dedicados a los terrores de la infancia (Bourke, 2005, p. 81-108) y a la segunda guerra mundial (Bourke, 2005, p. 222-254). Mención especial merece el libro de Alberti sobre la historia del corazón como centro en que se gestan las emociones, tanto a nivel de la cultura popular como en la historia de la medicina (Alberti, 2010). Utiliza, para llevarla a cabo, fuentes más o menos conocidas para los historiadores de la medicina, como son la autopsia del famoso cirujano inglés John Hunter (17281793) (Alberti, 2010, p. 41-60), o la autobiografía de la escritora y activista inglesa Harriet Martineau (18021876) (Alberti, 2010, p. 120-139). La lectura de ambos textos desde el prisma de la historia de las emociones, sin embargo, consigue encontrar nuevos datos relevantes allá donde otros habían descontado anécdotas. La historia de la angina de pecho o la importancia de ser diagnosticada con una enfermedad cardíaca en vez de uterina cobran nuevo significado gracias a la estupenda relectura de las fuentes realizada por Fay Bound Alberti.

No ha resultado, al menos hasta el momento, tan fructífera la localización de nuevas fuentes ${ }^{9}$. Todos los ejemplos señalados, y otros que podrían traerse a colación, basan sus excelentes resultados en la relectura de fuentes, pero ninguno, hasta lo que conocemos, ha intentado encontrar nuevas. Está claro que una par- 
te del problema reside en la dificultad de determinar qué puede ser una fuente para la historia de las emociones que no sean textos que hablen sobre emociones, como señala Rosenwein. Pero si aceptamos sin más esta situación nos encontraremos con casos, precisamente, como el de las comunidades emocionales de Rosenwein: la escasez de fuentes tradicionales (sean lápidas en cementerios o poemas cortesanos) produce una imagen de la vida emocional del pasado fragmentada, superficial y, en muchos casos, poco convincente. No se limita este problema a aquellos estudiosos de pasados remotos. Que las clases populares del siglo XIX han dejado pocas fuentes escritas de información (o que, en el caso de existir, son de dominio privado) es de sobra conocido. La historia de la medicina tiene su propia forma de aproximarse a este problema, que podemos identificar con lo que se ha llamado "perspectiva del paciente" (Porter, 1985). En el libro editado por Alberti encontramos, de hecho, notables ejemplos de este tipo de estudios, como el de Hillary Marland y su análisis del surgimiento de la "locura puerperal" en la Inglaterra del siglo XIX (Marland, 2006). El texto sigue tres líneas de investigación más o menos complementarias. Por un lado, el análisis de la evolución de los textos científicos sobre el tema a lo largo del siglo XIX; en segundo lugar, las prácticas médicas ejercidas sobre estas mujeres en los casos en que se producía su internamiento en instituciones psiquiátricas; en tercer y último lugar, el análisis de las prácticas de cuidado que se realizaban dentro del hogar de la enferma. En los tres casos, el foco se sitúa en la experiencia del paciente, ya sea a la hora de identificar su estado como una enfermedad, de aplicar un tratamiento o de estudiar el papel de los maridos en la recuperación de la esposa. Pero nuevamente nos encontramos con el mismo problema: las fuentes empleadas son, en su mayor parte, producidas por los médicos que las tratan: tratados de medicina, libros para el cuidado de enfermos en el hogar, casos médicos guardados en los archivos... todas las fuentes son producidas para estas madres "locas"; madres que nunca elaboran una narración de sus sentimientos, o que si lo hicieron no fueron conservadas. La utilización de estas fuentes, por muy lograda y excepcional que pueda llegar a ser $-y$ como son en la mayoría de los casos mencionados en este apartado-, deja una zona inexplorada, un gran blanco en el mapa emocional de las sociedades del pasado. Una zona habitada por aquellos que no nos han dejado fuentes escritas. En lo que respecta a las fuentes, la historia de las emociones debe ser capaz de encontrar fuentes alternativas que iluminen aspectos del pasado que hasta el momento han quedado "por debajo del radar".

\section{CONCLUSIONES}

La producción en historia de las emociones en los últimos diez años ha sido excepcional, tanto en la cantidad como en la calidad de la misma. En los últimos cinco años, además, se ha producido un proceso de institucionalización que ha dado lugar a la aparición de centros dedicados a su estudio en Londres, Berlín o Sidney, proyectos de investigación financiados por instituciones públicas y privadas, exhibiciones en museos de reconocido prestigio internacional, etc. Resulta obvio decir que la historia de las emociones ha evolucionado desde que Burke escribiera su texto en 2005. Sin embargo, como hemos visto, esta evolución en el reconocimiento institucional y público no viene emparejada con un desarrollo interno de la disciplina, que sigue trabajando en coordenadas similares a las detectadas por el padre de la nueva historia cultural. No obstante, el análisis que hemos realizado en estas páginas nos permite extraer ciertas líneas de fuerza que, desde nuestro punto de vista, conformarán la historia de las emociones en los próximos años, así como áreas en las que se requiere un mayor desarrollo.

La primera de estas líneas tiene que ver con el enfoque construccionista. Como resulta evidente por el análisis de los textos, se trata esta de la postura, convenientemente matizada, adoptada por la mayoría de los autores. Esta elección conlleva la realización de una historia cultural, tal y como avisaba Jordanova, que atienda a todos los aspectos de la vida humana. Este énfasis en la construcción de las emociones debe, sin embargo, ser complementado por una definición mínima, probablemente tentativa, de qué es una emoción, esto es, qué función cumplen las emociones en la vida del individuo. La propuesta de Reddy contiene algunos elementos que son interesantes en este punto, sobre todo su definición de la emoción como "percepciones" que no alcanzan a despertar nuestra "atención", pero que sí están "activadas" y, por tanto, afectan a nuestra relación con el entorno. Cómo afectan a esas relaciones, es decir, el "qué hacen las emociones" apuntado por Bourke, es un punto que debe tratarse en mayor profundidad, y que está estrechamente relacionado con el "quién" que ponía de manifiesto Rosenwein. Estas dos preguntas resultan fundamentales, ya que en ellas reside la historicidad de las emociones, sin que esto implique la existencia de un sustrato universal y biológico modificado culturalmente. Desde nuestro punto de vista, qué hace una emoción y quién es afectado son parte de lo que es una emoción. Detectar cambios en estos dos parámetros es detectar cambios en la emoción. La cólera del rey, por utilizar un ejemplo de Rosenwein, no es la misma cólera del campesino modulada culturalmente de forma distinta, son dos emociones diferentes(Rosenwein, 2006, p. 11; Althoff, 1998). La psicología social, extrañamente poco explorada por estos autores, puede ofrecer nuevas perspectivas desde las que desarrollar esta "definición mínima" (Tiedens y Leach, 2004). 
El segundo aspecto tiene que ver, en primer lugar, con la definición de la disciplina, y en última instancia deberá apoyarse en una teoría sobre la cultura, por muy tentativa que esta pueda ser. Desde nuestro punto de vista, y así nos parece entender la mayoría de los textos analizados, la historia de las emociones deberá apostar por un enfoque orientado a través del núcleo de la historia cultural, tal y como la define Mary Fissell: la atención al proceso de creación de significados (Fissell, 2004, p. 365). Una apuesta que empuja a huir de las "fotos fijas", al estilo de las comunidades emocionales de Rosenwein, para centrarse en los procesos, como señalaba Alberti. Para ello es necesario una teoría de la cultura que acentúe su carácter relacional y dinámico y que renuncie a la constitución de formas previas, como las de sujeto y objeto.

El tercer campo de actuación tiene que ver con la ampliación del alcance de la historia de las emociones hacia aquellos que, como hemos visto, no han dejado tras de sí evidencias escritas de su vida emocio-

\section{NOTAS}

1 'Our starting point is that 'the emotions', unchanging within human nature, transcending historical conditions, do not exist. Rather, 'emotions' are brought into being socially and historically [...]", Gouk y Hills, 2005a, p. 15.

2 Este grupo aparece, de forma más o menos consistente, en todos los artículos publicados desde el año 2000, hasta el punto de convertirse prácticamente en un listado "canónico" (Bourke, 2003; Rosenwein, 2002; Tausiet y Amelang, 2009; Matt, 2011). A este listado se unen, de forma más o menos habitual, representantes de la psicohistoria, sobre todo el estadounidense de origen alemán Peter Gay.

3 Alberti ha criticado que esta aproximación presupone un "yo emocional esencial" que, siendo influido por su contexto social, sin embargo lo prefigura. Desde su punto de vista, que compartimos, estas aproximaciones deberían tomar en cuenta los procesos en los cuales se produce el significado de las emociones (Alberti, 2006, p. Xvi).

4 El caso más evidente es el de Rosenwein. Pese a que en la introducción de su libro apuesta por una comprensión de las emociones como parte del proceso cognitivo, en la línea de Reddy o Martha Nussbaum, este componente cognitivo de las emociones no juega ningún papel en los estudios de caso contenidos en su libro (Rosenwein, 2006, p. 13). nal. Esto implica la necesidad de multiplicar nuestras fuentes, tanto a través de nuevas lecturas de fuentes conocidas o de la localización de nuevas fuentes textuales ${ }^{10}$ como del uso de otros tipos de fuentes cuyas posibilidades, hasta el momento, no han sido explotadas por la historia de las emociones, como pueden ser las fuentes visuales y las materiales.

\section{AGRADECIMIENTOS}

El presente trabajo se ha podido realizar gracias a una Beca para la Formación de Investigadores, modalidad predoctoral, del Departamento de Educación, Universidades e Investigación del Gobierno Vasco, ref. BFI07.33, dentro de los Proyecto de Investigación «Epistemología Histórica; estilos emocionales en los siglos XIX y XX» (FFI2010-20876), financiado por el Ministerio de Ciencia e Innovación, y «CREP-CM - Estudios de Ciencia y Cultura: Culturas, Espacios, Representaciones y Prácticas», financiado por la Comunidad de Madrid.

5 Vemos en esta definición, al igual que en Reddy y Stearns, el énfasis en el emotional management que, dice Burke, caracteriza a aquellos que se decantan por una posición más naturalista.

6 Rosenwein plantea varios conjuntos de comunidades emocionales que contienen, a su vez, subconjuntos. El ejemplo, en su caso, sería una comunidad emocional que podríamos identificar como "cristiandad" compuesta por pequeños subconjuntos como los de las comunidades de Trier, que comparten ciertas características pero se diferencian en otras. La comunidad mayor convive, a su vez, con otras que se componen, igualmente, de diversas comunidades menores (Rosenwein, 2006, p. 24).

7 El texto citado por Rosenwein es Stock, 1983.

8 Este análisis está recogido y ampliado respecto a lo presentado en el libro en el ya citado Bourke, 2003.

9 Nuevamente Bourke es una excepción, a través de la localización de escritos de pacientes o memorias autoeditadas.

10 Como el excelente trabajo llevado a cabo desde la Red de Archivos e Investigadores de la Escritura Popular, http://www2.uah. es/siece/red/aiep.htm. 


\section{BIBLIOGRAFÍA}

Alberti, Fay Bound (2006a), "Introduction: Emotion Theory and Medical History". En: Alberti, Fay Bound (coord.), Medicine, Emotion and Disease, 1700-1950, Basingstoke, Palgrave Macmillan, pp. xiii-xxvii.

Alberti, Fay Bound (ed.) (2006b), Medicine, Emotion and Disease, 1700-1950, Basingstoke, Palgrave Macmillan.

Alberti, Fay Bound (2010), Matters of the Hearth. History, Medicine, and Emotion, New York \& Oxford, Oxford University Press.

Althoff, Gerd (1998), "Ira Regis: Prolegomena to a History of Royal Anger". En: Rosenwein, Barbara H. (coord.), Anger's Past: The Social Uses of an Emotion in the Middle Ages, Ithaca \& London, Cornell University Press, pp. 59-76.

Battersby, Ch. (2005), "The Man of Passion: Emotion, Philosophy and Sexual Difference". En: Gouk, Penelope y Hills, Helen (2005), Representing Emotions: New Connections in the Histories of Art, Music and Medicine, Aldershot, Ashgate, pp. 139-153.

Bourke, Joanna (2003), "Fear and Anxiety: Writing About Emotions in Modern History", History Workshop Journal, 55 (1), pp. 113-16.

Bourke, Joanna (2005), Fear : A Cultural History, London, Virago Press.

Burke, Peter (2003), “Obertura: La nueva historia, su pasado y su futuro". En: Burke, Peter (ed.), Formas de hacer historia, Madrid, Alianza, pp. 13-38

Burke, Peter (2005), "Is There a Cultural History of the Emotions?" En: Gouk, Penelope y Hills, Helen (2005), Representing Emotions: New Connections in the Histories of Art, Music and Medicine, Aldershot, Ashgate, pp. 35-48.

Burke, Peter (2006), ¿Qué Es La Historia Cultural?, Barcelona, Paidós.

Derrida, Jacques (1976), Of Grammatology, Baltimore \& London, Johns Hopkins University Press.

Dixon, Thomas (2003), From Passions to Emotions: The Creation of a Secular Psychological Category, Cambridge, Cambridge University Press.

Dixon, Thomas (2006), "Patients and Passions: Languages of Medicine and Emotion, 1789-1850". En: Alberti, Fay Bound (coord.), Medicine, Emotion and Disease, 1700-1950, Basingstoke, Palgrave Macmillan, pp. 22-52.

Dror, Otniel E. (2005), "Dangerous Liaisons: Science, Amusement and the Civilizing Process". En: Gouk, Penelope y Hills, Helen (2005), Representing Emotions: New Connections in the Histories of Art, Music and Medicine, Aldershot, Ashgate, pp. 223-234.

Fissell, Mary E. (2004), "Making Meaning from the Margins. The New Cultural History of Medicine". En: Huisman, Frank y Warner, John H. (eds.), Locating Medical History: The Stories and Their Meanings, Baltimore \& London, The Johns Hopkins University Press, pp. 364-389.

Fleck, Ludwik (1986), La génesis y el desarrollo de un hecho cientifico, Madrid, Alianza Editorial.
Gouk, Penelope y Hills, Helen (2005a), "Towards Histories of Emotions". En: Gouk, Penelope y Hills, Helen (2005), Representing Emotions: New Connections in the Histories of Art, Music and Medicine, Aldershot, Ashgate, pp. 15-34.

Gouk, Penelope y Hills, Helen (2005b), Representing Emotions: New Connections in the Histories of Art, Music and Medicine, Aldershot, Ashgate.

Hayward, Rhodri (2006), "From Clever Hans to Michael Balint: Emotion, Influence and Unconscious in British Medical Practice". En: Alberti, Fay Bound (coord.), Medicine, Emotion and Disease, 1700-1950, Basingstoke, Palgrave Macmillan, pp. 144-168.

Hills, Helen (2005), “Architechture and Affect: Leon Battista Alberti and Edification". En: Gouk, Penelope y Hills, Helen (2005), Representing Emotions: New Connections in the Histories of Art, Music and Medicine, Aldershot, Ashgate, pp. 89-107.

Jordanova, Ludmilla (2004), "The Social Construction of Medical Knowledge". En: Huisman, Frank y Warner, John H. (eds.), Locating Medical History: The Stories and Their Meanings, Baltimore \& London, The Johns Hopkins University Press, pp. 338-363.

Labanyi, Jo (2010), “Doing Things: Emotions, Affect, and Materiality", Journal of Spanish Cultural Studies 11 (3), pp. 223-233.

Marland, Hilary (2006), Languages and Landscapes of Emotion: Motherhood and Puerperal Insanity in the Nineteenth Century. En: Alberti, Fay Bound (coord.), Medicine, Emotion and Disease, 1700-1950, Basingstoke, Palgrave Macmillan, pp. 53-78.

Matt, Susan J. (2011), "Current Emotion Research in History: Or, Doing History from the Inside Out", Emotion Review, 3 (1), pp. 117-124.

Nye, Robert A. (2003), "William M. Reddy, the Navigation of Feeling: A Framework for the History of Emotions", The Journal of Modern History, 75 (4), pp. 920-923.

Porter, Roy (1985), "The Patient's View: Doing Medical History from below", Theory and Society, 14 (2), pp. 175-198.

Reddy, William M. (2001), The Navigation of Feeling. A Framework for the History of Emotions, Cambridge \& New York, Cambridge University Press.

Richards, Graham (2005), "Emotions into Words - or Words into Emotions?". En: Gouk, Penelope y Hills, Helen (2005), Representing Emotions: New Connections in the Histories of Art, Music and Medicine, Aldershot, Ashgate, pp. 50-51.

Rosenwein, Barbara H. (2002), "Worryng About Emotions in History", The American Historical Review, 107 (3), pp. 821-28.

Rosenwein, Barbara H. (2006), Emotional Communities in the Early Middle Ages, Ithaca \& London, Cornell University Press.

Rosenwein, Barbara H. (2007), "Letter to the Editor", The American Historical Review, 112 (4).

Rosenwein, Barbara H. (2010), "Problems and Methods in the History of Emotions", Passions in Context 1 (I), pp. 1-32, [en 
línea], disponible en: http://www.passionsincontext.de/index. php?id=557 [consultado el 14/02/2013]

Schwartz, Michael (2005), "Bodies of Self-Trascendence: The Spirit of Affect in Giotto and Piero". En: Gouk, Penelope y Hills, Helen (2005), Representing Emotions: New Connections in the Histories of Art, Music and Medicine, Aldershot, Ashgate, pp. 69-87.

Stearns, Peter N. (1994), American Cool: Constructing a TwentiethCentury Emotional Style, New York, New York University Press.

Stock, Brian (1983), The Implications of Literacy: Written Language and Models of Interpretation in the Eleventh and Twelfth Centuries, Princeton, Princeton University Press.
Tausiet, María y Amelang, James S. (2009), "Introducción: Las emociones en la historia". En: Tausiet, María y Amelang, James (eds.), Accidentes del alma. Las emociones en la edad moderna, Madrid, Abada editores.

Tiedens, Larissa Z. y Leach, Colin W. (eds.) (2004), The Social Life of Emotions, Cambridge, Cambridge University Press.

Wickberg, Daniel (2007), "What Is the History of Sensibilities? On Cultural Histories, Old and New", American Historical Review $112(3)$, p. 661-684 